\title{
Factores étnicos culturales asociados a la lactancia materna en madres gestantes de Imbabura - Ecuador
}

\author{
José Hidrobo Guzmán \\ jfhidrobo@utn.edu.ec \\ Darwin Jaramillo Villarruel \\ Maritza Álvarez Moreno \\ Tatiana VásquezFigueroa \\ Yu Ling Reascos Paredes \\ Katty Cabascango Cabascango ${ }^{1}$
}

Facultad de Ciencias de la Salud, Universidad Técnica del Norte - Ibarra (Imbabura, Ecuador)

\section{RESUMEN}

Objetivo principal: Determinar las condiciones sociodemográficas asociadas a la lactancia materna en madres gestantes con enfoque étnico cultural. Materiales y métodos: Es un estudio cuantitativo, descriptivo y transversal, el instrumento de recolección fue aplicado en las diferentes Unidades Operativas de Salud de la Provincia de Imbabura Ecuador, corresponde a los cantones de Ibarra, Otavalo, Antonio Ante y Cotacachi. La población estudiada son 323 mujeres en estado de gestación, a partir de la semana 21 de embarazo, que acuden a sus controles prenatales. Resultados principales: La mayoría de mujeres gestantes son amas de casa y tienen el apoyo conyugal, respecto a la etnia, la mayoría se identifican como indígenasy mestizas, en cuanto al nivel de instrucción, tienen estudios de primaria y secundaria incompleta, y pocas son profesionales. Conclusión Principal: El nivel de conocimiento depende del nivel de instrucción por lo que este grupo presenta ideas diversas, con conocimientos generales sobre la lactancia materna como: alimentación exclusiva con leche materna, alimentación complementaria, alimentación a libre demanda, la manera correcta de colocar la mano de la madre en el pecho, desconocimiento sobre el calostro, frecuencia de lactancia materna, técnica y pociones de amamantamiento.

Palabras clave: lactancia materna exclusiva; factores asociados; madres gestantes. 


\title{
Cultural ethnic factors associated with breastfeeding inpregnant mothers of Imbabura - Ecuador
}

\begin{abstract}
Main objective: To determine the sociodemographic conditions associated with breastfeeding in pregnant mothers with an ethnic-cultural focus. Materials and methods: It is a quantitative, descriptive and cross-sectional study, the collection instrument was applied in the different Health Operative Units of the Province of Imbabura - Ecuador, it corresponds to the cantons of Ibarra, Otavalo, Antonio Ante and Cotacachi. The studied population is 323 pregnant women, from the 21st week of pregnancy, who come to their prenatal checkups. Main results: Most pregnant women are housewives and have spousal support, regarding ethnicity, most identify as indigenous and mestizo, in terms of educational level, have incomplete primary and secondary education, and few are professionals. Main Conclusion: The level of knowledge depends on the level of instruction, so this group presents diverse ideas, with general knowledge about breastfeeding, such as: exclusive feeding with breast milk, complement ary feeding, feeding on demand, the correct way to place the mother's hand on the breast, unawareness of colostrum, frequency of breastfeeding, technique and breastfeeding potions.
\end{abstract}

Key words: exclusive breastfeeding; associated factors; ethnic cultural factors; pregnant mothers.

Artículo recibido: 02 noviembre. 2021 Aceptado para publicación: 28 noviembre 2021

Correspondencia: jfhidrobo@utn.edu.ec Conflictos de Interés: Ninguna que declarar 


\section{INTRODUCCIÓN}

La Organización Mundial de la Salud (OMS), la Academia Americana de Pediatría (AAP) y el Comité de Lactancia de la Asociación Española de Pediatría, recomiendan la alimentación exclusiva del pecho durante los primeros seis meses de vida del niño y continuar con el amamantamiento junto con otros alimentos que complementen la alimentación hasta los dos años o más, mientras madre e hijo lo deseen. La falta de procedimientos centrados en el soportea la madre, así como las prácticas que la dificultan y reducen la producción láctea de la madre, influyen en el abandono precoz o fracaso de la lactancia materna. A nivel mundial, solo un $40 \%$ de los lactantes menores de seis meses reciben leche materna como alimentación exclusiva (1).

La Organización Mundial para la Salud (OMS) promueve activamente la lactancia natural como la mejor forma de nutrición para los lactantes y niños pequeños ${ }^{(2)}$. La lactancia exclusivamente materna en los primeros seis meses de vida aporta muchos beneficios al niño y a la madre, entre ellos se destaca la protección frente a las infecciones gastrointestinales, que se observa no solo en los países en desarrollo, sino también en los países industrializados (3) (4). Sin embargo, en ocasiones esta diversidad cultural afecta de alguna manera a todos los sectores de nuestra sociedad, incluida la sanidad (5). La lactancia materna, además de ser un acontecimiento biológico, es un hecho biográfico, ligado a las creencias y cultura de las mujeres, durante el cual, son muchas las experiencias vividas (6) (7). Está influenciada directamente por el medio en que se desenvuelve la madre y su rol en la sociedad cambia y altera el porcentaje de lactantes que se alimentan con leche materna $(\mathbf{8})$.

Se debe reconocer a la lactancia como una práctica necesaria y cualificada para construir una sociedad más saludable, más libre, más solidaria y más plena, por cuanto aporta significativamente a la disminución de la mortalidad y de la morbilidad infantil, evitable por desnutrición (9).

Existen diferentes prácticas y costumbres que se presentan de acuerdo con la cultura, creencias y etnia de las madres lactantes; estudios realizados en la provincia de Imbabura - Ecuador demuestran que existen madres indígenas que dan otro tipo de alimentos a sus niños antes de los seis primeros meses de vida, en base a las creencias que tiene la familia, como coladas hechas con leche, argumentando que esta tiene más nutrientes, además, se 
identifica que se da el seno materno hasta los tres años por creencias que la leche materna es un método de anticoncepción (10) (11).

Según investigaciones, del Fondo de Naciones Unidas para la Infancia, la consejería brindada a las madres por parte del personal de salud, mejora eficazmente las tasas de iniciación, duración y exclusividad de la lactancia materna en los países de ingresos bajos y medios, el apoyo de dicho personal por medio de consejerías reduce aproximadamente un $30 \%$ el riesgo de interrumpir la lactancia materna exclusiva (12) (13).

El objetivo principal de la presente investigación fue determinar las condiciones sociodemográficas asociadas a la lactancia materna en madres gestantes de la provincia de Imbabura - Ecuador con enfoque étnico cultural. Además, se identificaron los datos ginecológicos y se valoró el nivel de conocimiento que tienen las madres mestizas, indígenas y afro descendientes analizadas.

El estudio permite determinar el tiempo de duración de la lactancia materna exclusiva adoptadas en las diferentes etnias investigadas de acuerdo con variables de edad, con la finalidad de proponer la ejecución de intervenciones educativas que fomenten y mejoren los hábitos de lactancia materna de acuerdo con la población determinadas en el estudio.

El conocimiento sobre los beneficios de la leche materna y las técnicas de amamantamiento se convierten en un factor primordial para el mantenimiento de la lactancia dentro de los primeros seis meses de vida, junto a la condición sociodemográfica de las madres contribuirá $\mathrm{n}$ al desarrollo óptimo y a la preservación de la lactancia materna exclusiva, con estos antecedentes el estudio tiene como objetivo valorar los conocimientos que poseen las madres gestantes de la provincia de Imbabura que acuden a los controles prenatales en las diferentes unidades de salud, permitiendo obtener datos reales que contribuyan aplicar estrategias en beneficio de la madre y el niño.

\section{MATERIALES Y MÉTODOS}

Es un estudio cuantitativo, descriptivo y transversal, la recolección de datos permite realizarla medición numérica, con el fin de establecer el comportamiento de la población en estudio,a través de instrumentos validados con criterio de expertos y la utilización del paquete estadístico SPSS para el análisis de resultados de carácter descriptivos de frecuencias y cruce de categorías o niveles de una o más variables de las encuestadas con un enfoque étnico cultural. La investigación establece un tiempo y espacio determinados, 
para la exposición de resultado en la población definida.

Su estudio explicativo va más allá de la descripción de conceptos o fenómenos, están dirigidos a responder a las causas de la interrupción de la lactancia materna, cuales son los eventos físicos o sociales, y explica por qué ocurre este fenómeno y qué relación existe con otras variables: edad, condición étnica, estado civil, etc.

El instrumento de recolección de datos fue la encuesta, estructurada con preguntas normalizadas y dirigidas a una muestra representativa de la población, cuyo diseño y aplicación permite generar los datos necesarios de las diferentes Unidades Operativas de Salud de la provincia de Imbabura - Ecuador, en los cantones de Ibarra, Otavalo, Antonio Ante y Cotacachi, para alcanzar las propuestas de la investigación, con el fin dar a conocer las condiciones sociodemográficas, los datos ginecológicos y medir el nivel de conocimiento de las participantes sobre lactancia materna. El cuestionario permite estandarizar e integrar el proceso de recopilación de datos.

La población estudiada son 323 mujeres en estado de gestación, a partir de la semana 21 de embarazo, a través de la captación de mujeres embarazadas durante sus controles prenatales y reuniones del "Club de Embarazadas", "Club de la Mujer" y de "Lactancia Materna" en las diferentes Unidades Operativas de Salud, con la finalidad de fomentar de manera efectiva la lactancia materna exclusiva durante los seis primeros meses de vida del niño. Se aplica como criterios de inclusión y exclusión a quienes manifiesten por escrito su deseo de participar en el estudio mediante el consentimiento informado.

\section{RESULTADOS Y DISCUSIÓN}

\begin{tabular}{|c|c|c|c|}
\hline & & Frecuencia & Porcentaje \\
\hline \multirow{3}{*}{ Rangos de edad } & Menores de 20 años & 75 & $23,2 \%$ \\
\hline & De 21 a 34 años & 216 & $66,9 \%$ \\
\hline & Mayores de 35 años & 32 & $9,9 \%$ \\
\hline \multirow{5}{*}{ Rangos de edad } & Soltera & 97 & $30,0 \%$ \\
\hline & Casada & 135 & $41,8 \%$ \\
\hline & Divorciada & 5 & $1,5 \%$ \\
\hline & Unión libre & 85 & $26,3 \%$ \\
\hline & Viuda & 1 & $0,3 \%$ \\
\hline \multirow{6}{*}{ Auto identificación étnica } & Blanca & 4 & $1,2 \%$ \\
\hline & Mestiza & 137 & $42,4 \%$ \\
\hline & Indígena & 172 & $53,3 \%$ \\
\hline & Afrodescendiente & 2 & $0,6 \%$ \\
\hline & Afro ecuatoriana & 5 & $1,5 \%$ \\
\hline & Otras & 3 & $0,9 \%$ \\
\hline
\end{tabular}




\begin{tabular}{llcc}
\hline & Ninguno & $\mathbf{6}$ & $\mathbf{1 , 9 \%}$ \\
\cline { 2 - 4 } & Primaria incompleta & 19 & $5,9 \%$ \\
\cline { 2 - 4 } Nivel de instrucción & Primaria & 59 & $18,3 \%$ \\
\cline { 2 - 4 } & Secundaria incompleta & 58 & $17,9 \%$ \\
\cline { 2 - 4 } & Secundaria & 125 & $38,7 \%$ \\
\cline { 2 - 4 } & Superior incompleto & 26 & $8,0 \%$ \\
\cline { 2 - 4 } & Superior & 30 & $9,3 \%$ \\
\hline Total & $\mathbf{3 2 3}$ & $\mathbf{1 0 0 , 0 \%}$ \\
\hline
\end{tabular}

La mayoría de la población encuestada, el 66,9\% se encuentra entre 21 a 34 años, seguido del 23,2\% de mujeres menores a 20 años, datos que reflejan que la mayoría de mujeres gestantes se encuentran en una edad favorable para la concepción, además, se evidencia que existe un porcentaje muy significativo de las participantes que serán madres a muy temprana edad. Se determina de acuerdo con el estado civil que, el 41,8\% de la población son mujeres casadas, seguido de madres solteras con un $30 \%$ y un $26,3 \%$ que tienen unión libre (Tabla 1).

Estos datos permiten considerar que más de la mitad de las mujeres gestantes tienen el apoyo conyugal, situación que favorece a la adopción de rol materno según la teoría de Ramona Mercer, dicha teoría, permite incluir favorablemente a la pareja en la relación madre e hijo, y alcanzar la sensibilidad, empatía, actitud, y seguridad para ayudar a difuminar la tensión existente en la dualidad madre-hijo; factor que además disminuye en la mayoría de los casos la ausencia económica familiar, y se convierte en un componente que coadyuva al decremento de las actividades laborales de las madres en periodo de lactancia y aseguran el bienestar y desarrollo de los niños lactantes.

Por otra parte, se puede observar que existe un 53,3\% de la población que se identifica como indígena, seguido de un $42,4 \%$ de etnia mestiza (Tabla 1).

Dicha información recolectada determina una diferencia y permanencia de costumbres entre las dos etnias más representativas del estudio, y se manifiestan en poseer diferentes tipos de cosmovisión sobre la lactancia. En cuanto al nivel de instrucción, se observa que la mayoría de las mujeres embarazadas tienen estudios de primaria y secundaria, mientras que un $8 \%$ tiene educación superior incompleta y apenas un 9,3\% es profesional.

Patricia Hill Collins, señala cómo la experiencia subjetiva a la maternidad, la que se encuentra íntimamente ligada a los aspectos socioculturales de las comunidades étnico raciales a las que pertenecen, dimensiones que habían sido ignoradas desde la teorización feminista ${ }^{\mathbf{1 4}}$ ) 
Desde la Antropología se iniciaron reflexiones respecto a la interacción existente entre los distintos tipos de diferencia social y su efecto sobre el control de la capacidad reproductiva de las mujeres (15).

\section{Tabla 2: Datos ginecológicos}

\begin{tabular}{|c|c|c|c|}
\hline & & Frecuencia & Porcentaje \\
\hline \multirow{3}{*}{ Número de hijos: } & 0 & 138 & $42,7 \mathrm{t} \%$ \\
\hline & 1 & 131 & $40,6 \%$ \\
\hline & $>2$ & 54 & $16,7 \%$ \\
\hline \multirow{3}{*}{ Número de embarazos: } & 1 & 138 & $42,7 \%$ \\
\hline & 2 & 117 & $36,2 \%$ \\
\hline & $>3$ & 68 & $21,1 \%$ \\
\hline \multirow{3}{*}{ Partos normales } & 0 & 146 & $45,25 \%$ \\
\hline & 1 & 102 & $31,6 \%$ \\
\hline & $>2$ & 75 & $23,2 \%$ \\
\hline \multirow{3}{*}{ Cesáreas } & 0 & 289 & $89,5 \%$ \\
\hline & 1 & 21 & $6,5 \%$ \\
\hline & $>2$ & 13 & $4,0 \%$ \\
\hline \multirow{5}{*}{ Abortos } & 0 & 278 & $86,1 \%$ \\
\hline & 1 & 42 & $13,0 \%$ \\
\hline & 2 & 2 & $0,6 \%$ \\
\hline & 3 & 1 & $0,3 \%$ \\
\hline & Total & 323 & $100,0 \%$ \\
\hline
\end{tabular}

Los datos ginecológicos obtenidos en la encuesta, determinan que la mayoría son mujeres primigestas que representan el $42,7 \%$, el $40,6 \%$ tiene un hijo y el $16,7 \%$ restante son mujeres multíparas, datos que presuponen que las familias actuales se focalizan en concebir hasta 2 hijos y que son pocas las mujeres que optan por tener más de dos hijos. En cuanto al número de embarazos, el 78,9\% presentan de 1 a 2 gestas, seguido de un $21,1 \%$ con 3 gestas en adelante. que el $54,8 \%$ de las encuestadas presentan de 1 a 2 partos naturales, un 10,5\% manifestó haber presentado cesáreas y un 13,9\% abortos por diversas causas.

Al presentar más de la mitad de la población del estudio una paridad previa llevada a cabo, se deduce la relación de éste hecho con el conocimiento que poseían sobre la lactancia materna exclusiva. El parto medicalizado implica un buen número de intervenciones y manipulaciones no exentas de efectos colaterales para la madre y el bebé, entre los que se 
cuentan distintas secuelas físicas dolorosas en el posparto que pueden entorpecer la lactancia materna ${ }^{(\mathbf{1 6})}$. No tiene nada que ver empezar una lactancia después de un parto natural, libre de cables, sondas y sueros. Está también comprobado que las ingurgitaciones más importantes suceden a mujeres que han recibido sueros intravenosos y peridural. A grandes rasgos, cuanto más indemne salgas de tu parto, más fácil lo tendrás para iniciar la lactancia materna $(\mathbf{1 7})$.

Tabla 3

3.1. Rangos de edad vs ¿Cuánto tiempo puede dar leche materna sin otros alimentos-líquidos?

\begin{tabular}{|c|c|c|c|c|c|c|c|}
\hline & & 3 meses & 4 meses & 6 meses & 9 meses & 12 meses & Total \\
\hline \multirow{3}{*}{$\begin{array}{l}\text { Rangos } \\
\text { de edad }\end{array}$} & 15 años o menos & $14,3 \%$ & $14,3 \%$ & $42,9 \%$ & $28,6 \%$ & & $100,0 \%$ \\
\hline & De 16 a 35 años & $8,2 \%$ & $12,0 \%$ & $54,1 \%$ & $7,9 \%$ & $17,8 \%$ & $100,0 \%$ \\
\hline & 36 años o más & & $16,7 \%$ & $54,2 \%$ & $8,3 \%$ & $20,8 \%$ & $100,0 \%$ \\
\hline \multicolumn{2}{|l|}{ Total } & $7,7 \%$ & $12,4 \%$ & $53,9 \%$ & $8,4 \%$ & $17,6 \%$ & $100,0 \%$ \\
\hline \multicolumn{8}{|c|}{ 3.2. Nivel de instrucción vs ¿Qué es el calostro? } \\
\hline & & $\begin{array}{l}\text { Alimento de } \\
\text { la madre los } \\
\text { primeros dos } \\
\text { a tres días }\end{array}$ & $\begin{array}{l}\text { El nombre } \\
\text { de la leche } \\
\text { materna los } \\
\text { primeros } \\
\text { seis meses } \\
\end{array}$ & $\begin{array}{l}\text { La leche que } \\
\text { no tiene } \\
\text { defensas, ni } \\
\text { nutrientes }\end{array}$ & $\begin{array}{c}\text { Leche que } \\
\text { se da al } \\
\text { niño }\end{array}$ & Desconoce & \\
\hline \multirow{7}{*}{$\begin{array}{l}\text { Nivel de } \\
\text { instrucción }\end{array}$} & Ninguno & $33,3 \%$ & $50,0 \%$ & & & $16,7 \%$ & $100,0 \%$ \\
\hline & Primaria incompleta & $57,9 \%$ & $15,8 \%$ & & $5,3 \%$ & $21,1 \%$ & $100,0 \%$ \\
\hline & Primaria & $62,7 \%$ & $16,9 \%$ & & $1,7 \%$ & $18,6 \%$ & $100,0 \%$ \\
\hline & Secundaria incompleta & $56,1 \%$ & $14,0 \%$ & $1,8 \%$ & $3,5 \%$ & $24,6 \%$ & $100,0 \%$ \\
\hline & Secundaria & $64,8 \%$ & $10,4 \%$ & & $7,2 \%$ & $17,6 \%$ & $100,0 \%$ \\
\hline & Superior incompleto & $53,8 \%$ & $15,4 \%$ & & $3,8 \%$ & $26,9 \%$ & $100,0 \%$ \\
\hline & Superior & $63,3 \%$ & $13,3 \%$ & & & $23,3 \%$ & $100,0 \%$ \\
\hline \multicolumn{2}{|l|}{ Total } & $60,9 \%$ & $14,0 \%$ & $0,3 \%$ & $4,3 \%$ & $20,5 \%$ & $100,0 \%$ \\
\hline
\end{tabular}

3.3. Auto identificación étnica vs ¿Cada que tiempo se debe amamantar al lactante?

\begin{tabular}{|c|c|c|c|c|c|c|c|}
\hline & & Cada hora & $\begin{array}{c}\text { Cada tres } \\
\text { horas }\end{array}$ & $\begin{array}{l}\text { Cada vez } \\
\text { que llore }\end{array}$ & $\begin{array}{l}\text { Cada vez } \\
\text { que pida }\end{array}$ & Desconoce & \\
\hline \multirow{6}{*}{$\begin{array}{l}\text { Auto } \\
\text { identificaci } \\
\text { ón étnica }\end{array}$} & Blanca & & & & $50,0 \%$ & $50,0 \%$ & $100,0 \%$ \\
\hline & Mestiza & $16,1 \%$ & $18,2 \%$ & $13,1 \%$ & $36,5 \%$ & $16,1 \%$ & $100,0 \%$ \\
\hline & Indígena & $8,1 \%$ & $23,3 \%$ & $17,4 \%$ & $35,5 \%$ & $15,7 \%$ & $100,0 \%$ \\
\hline & Afrodescendiente & $50,0 \%$ & & $50,0 \%$ & & & $100,0 \%$ \\
\hline & Afroecuatoriano & & $40,0 \%$ & $40,0 \%$ & & $20,0 \%$ & $100,0 \%$ \\
\hline & Otras & & & & $100,0 \%$ & & $100,0 \%$ \\
\hline Total & & $11,5 \%$ & $20,7 \%$ & $15,8 \%$ & $35,9 \%$ & $16,1 \%$ & $100,0 \%$ \\
\hline
\end{tabular}


Los datos permiten realizar un análisis del tiempo que se debe alimentar al niño exclusivamente con leche materna, sin incorporar ningún otro tipo de alimento complementario incluido el agua, de ésta manera se observa, de acuerdo con los rangos de edad de las gestantes que, dentro del grupo con edad de 15 años o menos, el 42,9\% eligiero $n$ la respuesta correcta, es decir a los seis meses de edad del niño, al igual que en los grupos de 16 a 35 años y en el de 36 años o más, con un 54,1\% y un 54,2\%, respectivamente (Tabla 3 ).

En consecuencia, de acuerdo a los hallazgos encontrados, se determina que casi la mitad de la población investigada tiene conceptos erróneos en cuanto al tiempo específico recomendado para esta práctica de la lactancia materna exclusiva y permiten afirmar que aquellas madres que superan los 30 años de edad tienen más claro el concepto del tiempo específico de lactancia materna exclusiva, relacionando este hecho con la madurez y el número de hijos previos.

La lactancia materna presenta importantes beneficios a corto y a largo plazo tanto para el bebé que la recibe como para la madre que da el pecho y, de forma indirecta, para la sociedad abarcando ventajas de tipo nutricional, inmunológico, evolutivo, psicológico, social, económico e incluso ambiental.

La reducción de las tasas de lactancia materna se debe en parte al sistema sanitario, tanto los cuidados que recibe la madre durante su estancia hospitalaria como las características del lugar donde se produce el alumbramiento se relacionan con el inicio y la duración de la lactancia materna. La falta de procedimientos centrados en el soporte a la madre que da lactar, así como las prácticas que la dificultan y reducen la producción láctea de la madre, influyen en el abandono o fracaso de la lactancia materna exclusiva (2).

El conocimiento que tienen las gestantes sobre el calostro, de acuerdo a los datos obtenidos, demuestran que depende del nivel de instrucción, por cuanto se evidencio que las mujeres embarazadas que no tienen ninguna formación académica eligieron que el calostro es el nombre que recibe la leche materna los seis primeros meses de amamantamiento con un 50\%, mientras que las que tienen primaria incompleta optaron por el alimento que sale del pecho de la madre durante los primeros dos a tres días después del parto con un $57,9 \%$ y lo desconocen en un $21,1 \%$. Se pueden observar valores porcentuales muy importantes en gestantes que tienen secundaria y superior en cuanto a la selección de las opciones presentadas y que determinan el conocimiento del calostro 
según el nivel de instrucción. En cuanto al desconocimiento se presenta una media de 20,5\% en casi todos los niveles de instrucción (Tabla 3).

Esta información permite plantear necesariamente un proceso de Intervención Educativa que fomente la lactancia materna. La lactoferrina es una proteína presente en el calostro, con actividad antibacteriana y antifúngicas, aparece en la orina de los niños que lo han tomado, y parece ser que de esta manera el sistema urinario se protege también frente a las infecciones que suelen afectar a los recién nacidos con mucha facilidad (18).

El calostro, además, evita la adherencia de patógenos y reviste la mucosa gástrica de una capa protectora que evita que las bacterias puedan penetrar en ella. El calostro está lleno de células vivas que trabajan para proteger al recién nacido. Estas células transmiten información inmunológica de madre a hijo por lo que ofrecen una protección tanto a corto plazo como enla edad adulta.

El calostro tiene altas cantidades de sodio, potasio, cloruro y colesterol que tienen la función de estimular el crecimiento óptimo del corazón, el sistema nervioso centraly el cerebro. El calostro también tiene muchas más proteínas que la leche madura, que tienen una importante tarea de protección, nutrición y, además, controlan los niveles de azúcar en sangre del bebé. Esto es especialmente importante pues los bebés que tienen dificultades para mantener sus glicemias $\mathbf{( 1 8 )}$.

En cuanto a cada que tiempo se debe amamantar al lactante relacionadas con la auto identificación étnica, las gestantes mestizas afirman que se debe alimentar al niño cada vez que el/la niño/a lo pida, es decir a libre demanda con un 36,5\%, casi similar a lo que responde la etnia indígena, esto es un $35,5 \%$. Estos datos que se presentan determinan que existe un porcentaje superior al $75 \%$ de desconocimiento de las gestantes en todas las etnias investigadas, en lo que respecta a cada que tiempo se debe amamantar al niño (Tabla 3).

Estas respuestas demuestran que las madres gestantes no tienen claro la frecuencia en que se debe alimentar a su hijo, y expresan mayoritariamente ideas equívocas al responder horarios que no son correctas. Quienes respondieron alimentar al niño a libre demanda, están en lo correcto y manifiestan que el llanto es el único indicador de hambre. La OMS recomienda que a los seis meses (180 días) se empiece a dar a los lactantes alimentos complementarios, además de leche materna: 2-3 veces al día entre los 6 y 8 meses de edad, y 3 veces al día más un refrigerio nutritivo de los 9 a los 11 meses. 
Tabla 4

\begin{tabular}{|c|c|c|c|c|c|c|}
\hline \multicolumn{7}{|c|}{$\begin{array}{l}\text { 4.1. Auto identificación étnica vs ¿Cómo colocar correctamente la mano de la madre en el } \\
\text { pecho para el amamantamiento? }\end{array}$} \\
\hline & $\begin{array}{l}\text { Con la mano en } \\
\text { forma de } C\end{array}$ & $\begin{array}{l}\text { Con los dedos } \\
\text { en forma de } \\
\text { tijeras }\end{array}$ & $\begin{array}{c}\text { No } \\
\text { importa la } \\
\text { forma } \\
\text { sienta } \\
\text { cómoda }\end{array}$ & $\begin{array}{c}\text { No importa la } \\
\text { forma en la } \\
\text { que agarre la } \\
\text { mama }\end{array}$ & Desconoce & Total \\
\hline Blanca & $50,0 \%$ & $25,0 \%$ & & & $25,0 \%$ & $100,0 \%$ \\
\hline Mestiza & $38,7 \%$ & $35,8 \%$ & $15,3 \%$ & $7,3 \%$ & $2,9 \%$ & $100,0 \%$ \\
\hline Indígena & $48,3 \%$ & $29,1 \%$ & $11,6 \%$ & $5,8 \%$ & $5,2 \%$ & $100,0 \%$ \\
\hline Afrodescendiente & $50,0 \%$ & $50,0 \%$ & & & & $100,0 \%$ \\
\hline Afroecuatoriano & $20,0 \%$ & $60,0 \%$ & $20,0 \%$ & & & $100,0 \%$ \\
\hline Otras & $66,7 \%$ & & & & $33,3 \%$ & $100,0 \%$ \\
\hline Total & $44,0 \%$ & $32,2 \%$ & $13,0 \%$ & $6,2 \%$ & $4,6 \%$ & $100,0 \%$ \\
\hline \multicolumn{7}{|c|}{$\begin{array}{l}\text { 4.2. Auto identificación étnica vs ¿Qué estrategia educativa, le gustaría recibir para } \\
\text { mejorar los conocimientos sobre lactancia materna? }\end{array}$} \\
\hline & $\begin{array}{cc}\text { Charlas } \\
\text { educativas }\end{array}$ & Dramatizaciones & Talleres & $\begin{array}{c}\text { Discusiones } \\
\text { grupales }\end{array}$ & Otros & Total \\
\hline Blanca & $75,0 \%$ & & $25,0 \%$ & & & $100,0 \%$ \\
\hline Mestiza & $59,9 \%$ & $8,8 \%$ & $10,9 \%$ & $7,3 \%$ & $13,1 \%$ & $100,0 \%$ \\
\hline Indígena & $61,6 \%$ & $9,9 \%$ & $12,2 \%$ & $4,1 \%$ & $12,2 \%$ & $100,0 \%$ \\
\hline Afrodescendiente & $100,0 \%$ & & & & & $100,0 \%$ \\
\hline Afroecuatoriano & $100,0 \%$ & & & & & $100,0 \%$ \\
\hline Otras & $100,0 \%$ & & & & & $100,0 \%$ \\
\hline Total & $62,2 \%$ & $9,0 \%$ & $11,5 \%$ & $5,3 \%$ & $12,1 \%$ & $100,0 \%$ \\
\hline
\end{tabular}

Las madres gestantes de la etnia indígena mencionaron en un 48,3\% que la manera correcta de colocar la mano de la madre en el pecho para el amamantamiento es con la mano en forma de $\mathrm{C}$, seguido de un $29,1 \%$ de ellas que dijeron que lo harían en forma de tijera, mientras que las repuestas que se obtuvieron en la etnia mestiza en un 38,7\% en forma de C, y un $35,8 \%$ en forma de tijera. La información presentada refleja a un buen número de gestantes que manifiestan no tener importancia la forma en que se agarre la mama siempre que se sientan cómodas, lo que evidencia que la existe desconocimiento sobre la técnica correcta de agarre (Tabla 4).

Las estrategias educativas predilectas por las madres gestantes, para mejorar su nivel de conocimiento sobre temas de lactancia materna, mayoritariamente son las charlas educativas y talleres, seguido de las dramatizaciones, y un menor porcentaje las discusiones grupales (Tabla 4). En donde inicialmente predominó el bajo nivel de conocimiento, fue muy importante el impacto positivo que generan las estrategias educativas sobre lactancia materna, por cuanto, después de diversas intervenciones se 
evidenció un nivel alto de conocimiento, lo que demuestra la efectividad de la estrategia educativa, por el impacto logrado en la población intervenida.

La comprensión de la práctica de la lactancia materna y del proceso educativo que busca este estudio se hace desde el abordaje de las realidades sociales como objetos legítimos de conocimiento científico. Así, se parte de la interioridad de los sujetos participantes en la investigación para comprender las lógicas de pensamiento que guían las prácticas y acciones con respecto a la lactancia materna como resultado de un proceso histórico de construcción individual y colectivo. La modalidad del estudio fue la etnografía focalizada o particularista (16), dado que se realizó en una institución de salud específica, con madres gestantes y lactantes, sus familias y el personal de salud de la institución, lo que permitió una limitación geográfica y cultural (19).

\section{CONCLUSIONES}

La mayoría de la población en estudio se encuentra entre 21 a 34 años, con un porcentaje muy significativo de madres embarazadas a temprana edad; en donde predomina el estado civil de casadas y en menor proporción la unión libre, lo que permite considerar que más de la mitad de las mujeres gestantes tienen el apoyo conyugal; con respecto a la etnia, la mayoría se identifican como indígenas y mestizas; en cuanto al nivel de instrucción, tienen estudios de primaria y secundaria incompleta y, pocas son profesionales.

Las madres multíparas tienen de 1 a 2 hijos, y son pocas las mujeres que optan por tener más de 2 hijos, los partos son naturales, y muy pocas madres se les ha practicado cesáreas, los abortos en mínima cantidad por diversas causas.

El nivel de conocimiento, sobre la lactancia materna, depende del nivel de instrucción por lo que este grupo presenta ideas diversas y conceptos generales como: alimentación exclusiva con leche materna, alimentación complementaria a libre demanda, forma correcta de colocar la mano de la madre en el pecho, desconocimiento sobre el calostro, frecuencia de lactancia materna, técnica y posiciones de amamantamiento.

Las estrategias educativas elegidas por las madres gestantes, son las charlas educativas y talleres, seguido de las dramatizaciones y en un menor porcentaje las discusiones grupales. Se aplicó una variedad de estrategias y se implementó en algunas Unidades Operativas de Salud de la provincia de Imbabura, las mismas que ayudarán a mejorar el conocimiento a madres que sigan acudiendo a recibir atención (control prenatal y posparto) entre estas tenemos: banner, rompecabezas, gigantografías, carteles, imágenes ilustrativas, trípticos, 
hojas volantes, juegos formativos y didácticos.

\section{REFERENCIAS BIBLIOGRÁFICAS}

UNICEF. Lactancia Materna. [Online]. [cited 202005 29. Available from: https://www.unicef.org/ecuador/media/2611/file/Lactancia\%20materna.pdf.

OMS. Organización Mundial de la Salud. [Online].; 2017 [cited 2019 Enero 04. Available from:https://www.who.int/features/factfiles/breastfeeding/es/

OMS. https://www.who.int/topics/breastfeeding/es/. [Online].; 2020. Available from:https://www.who.int/topics/breastfeeding/es/.

OMS. [Online].; 2020. Available from: https://www.who.int/es/newsroom/fact-sheets/detail/infant-and-young-child-feeding.

Ma Dolores Gil-Estevan MdCSR. Diversidad cultural y lactancia materna. Prestación de cuidadosculturalmente competentes en Atención Primaria. Index de Enfermería. 2017.

M Dolores Gil-Estevan MdCSR. Diversidad cultural y lactancia materna. Prestación de cuidadosculturalmente competentes en Atención Primaria. Index de Enfermería. 2016;: p. $162-165$.

Talayero JMP. [Online].; 2004 [cited $2020 \quad 05 \quad 29 . \quad$ Available from:

https://www.ministeriodesalud.go.cr/gestores_en_salud/lactancia/CNLM_guia de_lactancia_mat erna_AEP.pdf

López-Sáleme R. Factores culturales y sociales asociados a la lactancia materna. Duazary. 2019;:p. 293 - 306.

Fdez-Vegue aG. Asociación Española de Pediatría. [Online].; 2013.

Available from: $\quad$ https://www.aeped.es/comite-nutricion-y-lactanciamaterna/lactancia- materna/documentos/lactancia-materna-demanda

Espinoza EP. Repositorio UTN. edu.ec. [Online].; 2016 [cited 2019 Julio 15. Available from:http://repositorio.utn.edu.ec/handle/123456789/6195.

OMS.

[Online].

Available

from:

https://apps.who.int/iris/bitstream/handle/10665/255731/WHO_NMH_NHD_14 .7_spa.pdf?ua=1

UNICEF. Fondo de las Nciones Unidas para la infancia. [Online].; 2013 [cited 2019 Febrero $7 . \quad$ Available from: http://www.unicef.org/spanish/nutrition/index_24824.html. 
MSP. [Online].; 2015. Available from: https://www.salud.gob.ec/wpcontent/uploads/2014/05/GPC-CPN-final-mayo-2016-DNN.pdf.

Saa TH. Análisis de la relación entre género y sexualidad a partir del estudio de la nueva divisióninternacional del trabajo femenino. Sociedad y Economía. 2014;: p. 213 $-238$.

Jordá DG, Bernal ZD. Perspectiva antropológica y de género en el análisis de la atención alembarazo, parto y puerperio. Revista Cubana de Salud Pública. 2010.

Materna AL. ¿Preparar la lactancia durante el embarazo? [Online].; 2019. Available from:https://albalactanciamaterna.org/.

Materna U. Lactancia Materna. [Online].; 2013. Available from: https://www.unicef.org/spanish/nutrition/index_24824.html.

Padró A. Laccapp. [Online].; 2018. Available from: https://blog.lactapp.es/el-calostro-untesoro/.

Nydia Stella Caicedo Martínez MCPDCOR. Educación para la lactancia materna Antioquia:Editorial Universidad de Antioquia; 2017 